\title{
Eliciting User Requirements with Older \\ Adults: Lessons from the Design of an \\ Interactive Domestic Alarm System
}

\author{
LORNA LINES
}

VIVID Research Centre

Department of Information Systems \& Computing

Brunel University, Uxbridge, UB8 3PH, UK.

Email Lorna.Lines@Brunel.ac.uk

Tel +44 (0)1895203397

Fax +44 (0)1895 251686

KATE S. HONE

VIVID Research Centre

Department of Information Systems \& Computing

Brunel University, Uxbridge, UB8 3PH, UK.

Category: Long paper

Keywords: Older Adults, Interactive Domestic Alarm Systems, User Requirement Capture, Focus Groups

\footnotetext{
Abstract: This paper documents how methodological challenges were addressed when identifying user requirements for an Interactive Domestic Alarm System (IDAS) designed to enable older adults to live independently in their own homes for longer. A novel approach to determine possible IDAS functionality is described, and the results of focus groups conducted with older adults and care workers are reported. The paper identifies some difficulties encountered when using the focus group method with an ageing sample, and highlights the importance of careful preparatory work if this method is to be used successfully in such a context.
} 


\section{Introduction}

The UK, in common with many Western societies, has an increasingly ageing population. Transformations in health, social and lifestyle trends have led to decreases in mortality and fertility rates and increases in life expectancy. As a result, this had led to a population forecast indicating that $30 \%$ of the UK population will be over the age of 60 by the year 2031 [2]. Although they may be a cause for celebration, these figures also present problems. A recent study revealed that local authorities across the UK reported concern about inadequate resources for the services they could (and could not) provide for older adults [4]. The expected increase in the aged population is likely to create greater demands on these already overburdened service providers. To alleviate pressure on housing and care services, it is likely that there will be a greater emphasis on assisting those eligible for services within their own homes [1]. Such assistance is likely to be favourable for both service providers and clients, as over $80 \%$ of older adults' consider their independence and living in their own home as very important [5]. The development of technologies to assist older adults with living independently in their own homes may provide a promising solution.

For this reason, smart home technologies are being utilised to develop Interactive Domestic Alarm Systems (IDASs) designed specifically to assist older adults with independent living.

The design of an IDAS is a good example of an area where the application of new technology has great potential to improve the lives of older adults. However, it is also an application area where designers must be particularly sensitive to the needs and requirements of users. An IDAS will be implemented within users' homes, they will be pervasive and they will potentially detect intimate details of users' lives. The highly personal nature of the human-computer relationship in this context means that achieving user acceptance of the technology is a high priority. IDAS development is thus an area in which effective gathering of user requirements is vital, and as it is a novel application area, there is also large scope for defining possible functionality. Given these features, IDAS development represents an ideal context in which to consider the elicitation of user requirements with older adults. 
This paper documents how methodological challenges were addressed when eliciting user requirement specifications for an IDAS for older adults. A novel, structured approach to determine possible functionality of a domestic technology is described, and the results of focus groups evaluating possible IDAS functionality with both older adults and key stakeholders are reported. The difficulties encountered when using the focus group method are discussed, raising the issue of the suitability of popular methods used in the design of interactive technologies when applied to an ageing sample. We use our experiences to propose ways in which the process of eliciting user requirements from older users can be made most effective. This provides the paper's main contribution to Universal Access, since in order to achieve Universal Access to technology it is vital that methods are found which can elicit requirements from all types of users. The paper proceeds as follows: in order to put the research into context, we begin by defining the concept of an IDAS in more detail; we then describe the process which was followed in eliciting user requirements and the results obtained; finally, we end by discussing the implications of our research.

\section{Interactive Domestic Alarm Systems}

An IDAS is a major departure from traditional 'detect and alert' domestic alarm systems such as fire and burglar alarms. Fire and burglar alarms are dedicated alarm systems for single alarm states, whereas an IDAS can detect a variety of alarm states that may have negative consequences for an older adult. These might include, for example, doors and windows left open, low room temperatures, or a fall of the older adult. To further the distinction, traditional domestic alarms provide limited possibilities for interaction with the user. IDASs aim to promote independent living by providing the opportunity for dialogue and negotiation between the user and the system. IDASs may, as do some current domestic alarm systems, initiate contact via telecommunication networks with external care agencies if the older adult is unable to resolve the alarm state.

To explain how an IDAS may operate consider for example the Millennium Home System, currently being developed through an academic and industrial collaboration based at Brunel University. This IDAS operates using sensors, retrofitted to the fabric of the older adults home, that detect changes in the domestic environment. Sensor data are monitored and analysed by a central 
computer that, upon detection of an alarm state, activates the user interface to alert the user to the alarm state. At this stage, it is expected that the user will resolve the alarm state. If the user resolves the alarm successfully, the system will resume a 'safe state'. However, if user feedback is not detected, either via direct manipulation of the environment (for example, closing a door) or via a dedicated input device, the central computer will initiate an alarm call, via a call centre, to an external care agency, requesting human assistance. A 'safe' system state will only be resumed when human assistance has been received. Differences between IDASs will lie in the specific technologies utilised and the number and type of alarm states that can be detected.

\section{IDAS User Requirement Capture: Methodological Challenges}

For human factors, a major challenge is how to define the functionality of a novel system of this kind, such that it meets the needs of both users and other stakeholders (carers, councils, insurers, etc.). Specifically, we need to address how we define user requirements in terms of the following key considerations:

- What events or situations the system should detect to maintain the health and safety of the home occupant;

- The system behaviour (e.g., outputs, human-computer dialogue) in response to the detection of given events or situations.

In this paper, the first of these considerations is addressed. The decision of whether to detect an event should be primarily influenced by the wishes of users. More pragmatically, the available technology will place limitations on what can be detected. The design will also need to meet the legal and ethical requirements of other stakeholders. While users, stakeholders and technological limitations should all play a role in selecting functionality, we argue that this is best done by using them to evaluate possible functionality, rather than to generate possible functionality. For example, when generating possible functionality, technology may be limited by the expectations of the users', stakeholders and system designers. The risks of technology-driven design are well known; conversely, the risks of allowing users to "invent" the functionality of the system are that important functions may be omitted, due to the complexity of the domain. This perspective led us to adopt the approach described below. We began with a 
structured analysis of the domain in order to define an exhaustive list of possible functionality. The results of this analysis were then used as the starting point to research involving potential system users and stakeholders.

\section{A Novel Approach: Human Reliability Assessment}

To identify the possible functionality of an IDAS to be evaluated by target users, a structured framework derived from Human Reliability Assessment (HRA) was used. HRA's generic goals are to identify, quantify and reduce human error [8]. HRA is largely used in high technology-high risk industrial settings. However, it is a suitable framework for a low technology domestic setting within which an IDAS will function, and promotes the idea of protecting human life and health. HRA is concerned with the elimination or reduction of human error, and thus becomes more compelling as an appropriate framework when we consider that the majority of risk situations in the home are a result of human error [13].

Two key methods were employed by the researchers to conduct the HRA. The first was Task Analysis (TA) that offers a structured approach for the identification of domestic tasks and the way in which they may be performed. In the case of an IDAS, the overall task of interest was 'independent living'. Each task was identified in terms of, a) the requirement fulfilled by the task, b) the required behaviour to conduct the task and c) the identification of the typical events that constitute the required behaviour. Following the TA of independent living, the method of Human Error Analysis (HEA) was conducted. HEA uses the output of the TA to identify the ways in which human behaviour could lead to negative outcomes for the user, in this specific case, the resident. A simple and effective approach is to identify External Error Modes (EEMs) [14] that describe human error at the level of simple, observable behaviours. To understand how these techniques were used, consider the following example of a TA for the task of consuming food. Food consumption is a task that occurs within the domestic environment. The physiological requirement fulfilled by this task is the intake of nutrients. The behaviour needed to meet this requirement is the regular consumption of food. Following the TA of food consumption, HEA identified the EEMs that could occur during task performance and the negative consequences that may arise as a result of omission errors (i.e., not performing the task) or errors 
of commission (i.e., performing the task too little, too often or inadequately). Based on the TA for food consumption, omission errors would comprise not consuming food and commission errors include consuming an inadequate amount). The negative consequences that may arise from these EEMs include weight gain, weight loss and starvation, the latter leading to fatal consequences. Following the TA and HEA, the time frame for concern, i.e., the amount of time before action was necessary to prevent a negative consequence for the user, was considered. For example, the negative consequences associated with the EEMs for food consumption give rise to a time frame of days for errors of omission and a time frame of days or weeks for errors of commission.

Upon completing the TA and HEA, a content analysis was conducted that generated a list of 26 domestic activities. Only those activities that, through errors of omission or commission, could generate negative consequences for the resident were included. These activities were considered as the possible events that could be detected by an IDAS. This list of activities, which is documented in the following section, was used as the starting point for focus group research involving potential users and stakeholders.

\section{A Popular Method: Focus Groups}

From a human factors perspective, it is crucial that the core user requirements of an IDAS are gathered in terms of which alarm states the target user group would or would not like to be detected. To gather the core user requirements, we decided, given the project's time limits, financial constraints and application domain, that the most appropriate research method was focus groups.

Recent work in the design of interactive systems suggests that focus groups are a method 'of considerable power, precision and innovation' [11]. Focus groups are a cheap, critically reflective and ecologically valid [3] means of gathering information from target users. As can be seen in the literature [for examples, see $9,10,12]$, a focus group comprises discussion moderators and, typically, between 5 and 12 target users. Moderators initiate the topics for discussion and prompt further elaboration of the issues where appropriate. The use of focus groups for the elicitation of IDAS user requirements became more compelling when it was noted that focus groups were considered appropriate for use with an ageing sample [see 7]. 
The following sections report three focus groups conducted with older adults and a focus group comprising care workers. The main aim of the focus groups was to elicit user requirement specification for IDAS functionality through the evaluation of the possible functionality of an IDAS included in the list of activities that could lead to negative consequences generated by the TA and HEA. Therefore the main objectives were to:

- Find out which alarm states should be detected by an IDAS

- Explore the reasons why particular alarm states should or should not be detected.

Each focus group followed the same procedure, however, due to difficulties experienced by the researchers, focus groups became increasingly more structured. The reasons for this, together with the lessons that can be learned, are discussed in detail in section 7.3. The same moderators oversaw each of the focus groups. Identical materials and general procedure were used throughout each of the four focus groups.

\subsection{Participants}

Focus group 1: 12 older adults [1 male, 11 female], all over the age of 65 and living independently.

Focus group 2: 5 older adults [1 male, 4 female], all over the age of 65 and living independently.

Focus group 3: 5 older adults [2 male, 3 female], all over the age of 65 and living independently.

Focus group 1 - 3: For safety, legal and ethical reasons, during the focus groups with older adults care workers from Hillingdon Social Services attended the sessions.

Focus Group 4: 6 care workers [1 male, 5 female] employed by Hillingdon Social Services, Home Care Service Department.

\subsection{Materials}

Tape recorder, audio tape, notebooks, pens, whiteboard, whiteboard markers, list of 26 activities that could be detected by an IDAS: (1) Food/Nutrient Intake; (2) 
Drink/Fluid Intake; (3) Sleep/Rest; (4) Personal Temperature Regulation; (5) House Temperature Regulation; (6) Elimination (Excretion); (7) Elimination (Urination); (8) Activity (Movement/Mobility); (9) Management of existing medical conditions; (10) Management of sudden onset medical conditions; (11) Management of slow onset medical conditions; (12) Financial management; (13) Monitor and maintain furniture, fixtures and fittings; (14) Monitor and maintain home appliances; (15) Monitor and maintain home utilities (e.g., gas and electricity supplies); (16) Home Security (monitoring of entry and exit points); (17) Personal Security (personal alarms and self defence); (18) Home Entertainment (e.g., TV, Hobbies, Reading); (19) Outside Entertainment (e.g., Outings and hobbies); (20) Communication; (21) Home Hygiene; (22) Personal Hygiene; (23) Food and drink storage; (24) Food and drink preparation; (25) Food and drink cooking/making; (26) Clothing Hygiene.

\subsection{The procedure}

The focus group participants were thanked for attending the session and informed of their ethical rights. They were advised that any information that they provided would remain anonymous, and that they could withdraw from the discussion at any point in time, should they wish to. The focus group moderators provided the participants with background information, explaining the motivation behind the development and general functionality of an IDAS. Following this short presentation, the participants were then informed that they were required to give advice on whether the activities that would be discussed should be detected by an IDAS. They were also informed that it would be very useful if they could explain the reasons as to why the activities should be detected. Subsequently, the moderators opened the discussion, starting with the first activity on the list, and continued to raise each activity in sequential order. The moderators elaborated on the activities where necessary, and when no further comments were forthcoming they would raise the next activity to be discussed. The focus groups were tape recorded for transcription and analysis purposes, and additional notes were taken documenting difficulties that were being experienced by the moderators. When all of the activities that could be detected by an IDAS had been considered and the discussion exhausted, the moderators informed the participants that the results of 
the focus groups would be available in due course, and thanked them once again for attending.

\section{Results}

\subsection{Focus groups $1-3$ (Older Adults)}

\subsubsection{Activities to be detected}

Responses to each activity that could be detected by an IDAS were recorded in terms of:

A) The older adults wanted the activity to be detected by an IDAS [Yes]

B) The older adults did not want the activity to be detected by an IDAS [No]

C) The older adults thought that in the right circumstances it could be useful for an activity to be detected by an IDAS [Maybe]

Table 1 below shows the overall results of the three focus groups revealing which of the 26 activities were chosen by all of the participating older adults as activities to be detected and those that participants considered as useful to detect in appropriate circumstances.

\section{Table 1}

Of the 26 activities deemed appropriate for IDAS detection, only 5 were considered as those that should be detected by an IDAS at all times (Personal temperature regulation, House temperature regulation, Management of existing medical conditions, Management of sudden onset medical conditions, home security and Personal security), whereas the remaining 3 activities (Activity, management of slow onset medical conditions and personal hygiene) are considered appropriate for IDAS detection only in suitable circumstances.

\subsubsection{Exploration of older adults' choice of activities to be detected}

The amount of information gained throughout the focus groups decreased as the focus groups became increasingly structured (discussed in detail in section 7.3). However, to summarise the observations made during the transcript analysis, 
older adults' were reluctant to have tasks of an intimate or personal nature, such as toilet habits or hygiene, detected. The more able-bodied older adults tended to view the detection of such activities as completely unnecessary. However, the more frail participants were more likely to accept that there may be situations where detection of these tasks would prove useful.

\subsection{Focus Group 4: Care workers}

Responses to each activity that could be detected by an IDAS were recorded in terms of:

A) The care workers wanted the activity to be detected by an IDAS [Yes]

B) The care workers did not want the activity to be detected by an IDAS [No]

C) The care workers thought that in appropriate circumstances it could be useful for an activity to be detected by an IDAS [Maybe]

Table 2 below shows the overall results of the focus group revealing which of the 26 activities were chosen by the participating care workers as activities to be detected and those that were considered as useful to detect in appropriate circumstances.

\section{Table 2}

All of the activities presented to the care workers were deemed appropriate for IDAS detection. The responses highlighted in table 2 are those that match the responses given by the older adults. 19 of the 26 activities were considered to be suitable for an IDAS to detect, whereas only 7 of the activities were considered suitable for detection in the right circumstances.

\subsubsection{Exploration of care workers' choice of activities to be detected:}

From analysis of the transcripts it is clear that the care workers approached the discussion in terms of how an IDAS may be useful to them. Specifically, the type of information that could be useful when making an 'on-the-scene' assessment of situations that had escalated to the level of a negative consequence for an older adult. For example, the care workers responded positively to the detection of the resident's death so that they could be forewarned of this distressing situation prior 
to attending. This is by no means a criticism of how care workers dealt with the discussion of the task, as all support for detection came from their own experiences of dealing with elderly clients in their own homes.

\section{Findings \& Discussion}

The following section reports the main findings from this investigation into the elicitation of IDAS user requirements. The differences between the desired functionality of an IDAS from the older adults' and care workers' perspectives are discussed. A detailed discussion is presented that considers the suitability of employing focus groups with older adults in the design and development of interactive systems. Finally, future directions for research are suggested.

\subsection{Main Findings: IDAS User Requirements}

Based on the results provided by both older adults and care workers, 9 user requirements of IDAS functionality were agreed upon throughout the four focus groups. These are:

1. Personal Temperature Regulation

2. House Temperature Regulation

3. Activity (Movement/Mobility)

4. Management of Existing Medical Conditions

5. Management of Sudden Onset Medical Conditions

6. Management of Slow Onset Med Conditions

7. Home Security (monitor entry \& exits points)

8. Personal Security (alarms \& self defence)

9. Personal Hygiene (e.g., hair, body, hands, teeth)

Exploring the reasons as to why an activity should be detected or not proved difficult with the three focus groups comprising older adults. They provided a large amount of unrelated information as they began to 'wander' from the specific activity being discussed. However, throughout the focus group transcripts, one theme dominates negative reactions to the detection of certain activities, namely, that older adults do not wish for personal or intimate activities to be detected. Perhaps this is due to a need for privacy, along with the desire for independence. With this in mind, it is not surprising that the activities associated with stereotypically taboo subjects such as elimination [i.e., excretion and urination] 
were also met with strong negative responses when it was suggested that these activities could be detected by an IDAS. However, it was noted that the more frail the older adult, the more likely they were to reconsider the detection of elimination when the benefits of monitoring bathroom behaviour was proposed. Such detection could indicate ill health and if detected at an early stage by an IDAS could lead to quicker resolution, should an external care agent be contacted. In contrast, the care workers delivered positive responses to the detection of all of the activities proposed for detection. All responses fell into the 'Yes' or 'Maybe' detection categories. The differences between the overwhelmingly positive responses of the care workers when compared to the older adults' could simply be due to differing personal perspectives. Both groups addressed the detection of activities from their own viewpoints. The older adults appreciated that they would be immediately affected should there be a problem with their performance of a particular activity. The care workers, on the other hand, dealt with the issue of detection in terms of how to avoid the negative consequences that they often deal with when activities fail to be conducted in an appropriate manner.

\subsection{Focus groups - A suitable method?}

As previously mentioned, throughout the focus groups conducted with older adults the moderators experienced a number of difficulties. It is suggested that these difficulties are related to the age of the sample, since these difficulties were not experienced when conducting the focus group with care workers, a group of young/middle aged adults. The fact that difficulties were experienced was a surprise given that it had been previously documented that focus groups are an appropriate method to be used with older adults with no need for modification [see 7]. However, since conducting this research other authors have also commented on experiencing difficulties when conducting focus groups with older adults [see 6]. Specifically, difficulties were encountered when attempting to manage focus groups comprising more than three older adults. In [6] it is suggested that these difficulties may have been due to auditory impairments and the older adults' ability to follow the discussion.

The problems experienced during the first focus group with older adults were related to keeping the discussion focussed on the activities that could be detected by an IDAS. The participants were inclined to 'wander' from the topic under 
discussion, providing instead unrelated anecdotes and chatting amongst themselves. It was difficult to keep the participants' attention focussed on the task. Whether these attention problems were due to the cognitive demands of the session or to fatigue or boredom is unclear. There were, however, two factors that we felt were contributing to the problem: the large number of participants (12) recruited for this particular session, and the loosely structured approach that the moderators had adopted.

As a result of the problems experienced in the first focus group, the second focus group employed a smaller number (5) of older adults. Some increased structure was also imposed by avoiding the use of overly broad, open-ended questions. There were some notable advantages in the smaller group size, for example the session was more productive in the sense that everyone had time to contribute, and those who appeared nervous could be drawn into the discussion more easily by the moderators. However, there were still problems with keeping the attention of the group focussed on the task.

Both the first and second focus groups were effective at drawing out user opinions on which activities should be detected by an IDAS, but were less effective at probing why activities should be detected. Notably, the discussion structure for the first of these objectives was closely based on the outcomes of the task analysis and human reliability assessment stage of the research. A looser structure was adopted for discussing why activities should be detected, since we had hoped that this would generate more in-depth explanations. Instead we found that, even with the less open-ended approach used in the second focus group, it was during these less structured discussions that the moderators had most problems in keeping the participants' attention. For the third and final focus group conducted with older adults, a full structure was imposed to probe both objectives. Here, as in the previous groups, the discussion of functionality was based on the list of activities generated during the human reliability assessment phase. In addition, in this focus group, the questions about why particular activities should be detected were replaced by a structured discussion around the need to detect particular categories of negative outcome for the home occupant. The categorisation used, based on both severity of negative outcome and timeframe for concern, had also been developed during the human reliability phase of our research. As a result of this amendment to the focus group procedure, less inter-group chatting was observed, 
and the topics were discussed more quickly than in previous sessions. In general, it was felt that IDAS user requirements were successfully obtained. However, the depth of information obtained was limited, with limited possibility of exploring the reasons behind the choices the target users had made.

\subsection{Possible Alternatives}

Given the difficulties experienced when keeping older adults focussed on the topic for discussion during the focus groups, other methods may have been more suitable to elicit the user requirements for the Millennium Home System. For more in-depth information, perhaps individual semi-structured or structured interviews would have been more appropriate. For a wide breadth of information from a larger number of target users, a series of focus groups with no more than three older adults may have provided similar data. However, both of these alternatives are more time consuming than conducting focus groups with larger numbers.

\section{Conclusions}

Our experience of user requirements elicitation in the context of IDAS development allows us to draw several conclusions. Focus groups with older adults require more careful handling than those with younger adults. We found that focus groups with older adults ran most smoothly when (a) a highly structured approach was used, and (b) a relatively small group of participants was involved. The strong need for structure in the focus groups meant that the preparatory work that we had done, using human reliability assessment to define possible system functionality, proved invaluable. This analytic research provided us with a clear structure around which to base discussions with participants. As the need for structure became increasingly clear, we progressively increased structuring in our approach. We argue that this preliminary analysis stage contributed significantly to our successful use of focus groups in this context. Consequently, we recommend that the use of focus groups in user requirements gathering for older users be preceded by a thorough analysis of the domain. The results of such an analysis should be used to design highly structured focus groups, each composed of relatively few participants. Such an approach is, we 
believe, the best way to exploit the potential benefits of using focus groups with older users.

We found the focus group method less effective with older users when the procedure was less structured, because of the problems of keeping the participants 'on topic'. For the same reason, it was difficult to obtain in-depth information during focus groups with older users. This suggests that there will be a number of situations where focus groups will not be a suitable method for requirements elicitation with older users. One such situation is where the research is highly speculative and little is known about the domain. To re-iterate the point made above, researchers need a good understanding of a domain in order to structure the focus group approach sufficiently for it to work well with this user group. Another situation where focus groups will be less suitable is where researchers are concerned with eliciting in-depth responses. In such situations it may be that interviews or even smaller groups may be a more effective way of interacting with potential users.

We recommend suitable alternatives to the focus group method for user requirement capture with older adults. Where detailed information is required we suggest that individual semi-structured or structured interviews may provide the necessary data. When there is a need for input from a larger sample of older adults, a series of focus groups, with no more than three participants, may prove successful. However, we note that although potentially suitable and likely to overcome the experienced difficulties associated with structure and keeping the older adults 'on-track', these methods may demand extra time resources. Finally, we noted from our research that the focus groups with older users and those with carers elicited noticeably different patterns of results. While the viewpoints of both groups are valid, this result does emphasise the need to include older users themselves in requirements specification work. Carers may be considered experts at dealing with the problems experienced by older adults, but this does not mean they will necessarily share the same concerns about the use of technology.

\subsection{Future directions}

Since this work was conducted, the IDAS user requirements have been implemented in the design and development of the Millennium Home System 
described earlier in this paper. Future research into user requirements of older adults for interactive system design needs to further investigate, and disseminate results concerning, issues related to the suitability of popular methodologies employed with an ageing sample. From the use of focus groups reported in this paper, it is clear that, at present, perhaps due to the limited research into the design of interactive technologies specifically for older adults, both older adults and researchers may be disadvantaged in terms of their use and design of technologies if popular methodologies are not adapted to meet the needs of an ageing user group.

\section{Acknowledgements}

This research was supported by the Millennium Home Project (EPSRC/DTI LINK Foresight/DoH Medlink). We would like to thank Hillingdon Social Services for their cooperation and assistance with participant recruitment.

\section{References}

[1] Dewsbury GA, Edge HM (2000) Designing the Home to Meet the Needs of Tomorrow...Today:

Deconstructing and rebuilding the home for life. Paper presented at the ENHR 2000 Conference, June 26-30,

Gävle

[2] Dunnell K (2000) Policy Responses to Population Ageing And Population Decline - United Kingdom.

Paper presented at the The Expert Group Meeting On Policy Responses To Population Ageing and Population Decline, Population Division, Department of Economic and Social Affairs, United Nations Secretariat, New York, US

[3] Elling R (1997) Revising safety instructions with focus groups. Journal of Business and Technical Communication, vol 11 pp451 - 468

[4] Help the Aged (2002a) Nothing Personal: Rationing Social Care for Older People. Available: http://www.helptheaged.org.uk/Viewpoint/Research/_default.htm.

[5] Help the Aged (2002b) The Older Population. Available: http://www.helptheaged.org.uk/AdviceInfo/InfoPoint/_default.htm.

[6] Inglis E, Szymkowiak A, Gregor P, Newell AF, Hine N, Wilson BA, Evans J (2002) Issues surrounding the user centred development of a new interactive memory aid. In: Keates S, Langdon P, Clarkson PJ, Robinson P (eds) Universal Access and Assistive Technology, pp171 - 178. Springer.

[7] Kirakawski J (1997) Methods for user-orientated requirements specification. Telematics Applications Project TE2010. Available: http://www.ejeisa.com/nectar/respect/3.2/index.htm

[8] Kirwin B (1991) Human Reliability Assessment. In: Wilson J R, Corlett EN (1991) Evaluation of Human Work. A practical ergonomics methodology, pp 706-754. Taylor \& Francis: London

[9] McDonagh-Philp D, Bruseberg A (2000) Using Focus Groups to Support New Product Development. Institute of Engineering Designers Journal, vol 26 (5) pp 4-9 
[10] Millward LJ (2000) Focus Groups. In: Breakwell GM, Hammond S, Fife-Schaw C (eds) Research Methods in Psychology (2ed) Sage. pp 303-324

[11] Rosenbaum S, Cockton G, Coyne K, Muller M, Rauch T (2002) Panel on Focus Groups in HCI: Wealth of Information or Waste of Resources? Proceedings of CHI 2002, pp702-703, April 20-25, Minneapolis, USA [12] Robson C (1993) Real World Research. Blackwell

[13] Royal Society for the Prevention of Accidents (2000) Home Safety: The RoSPA Guide to Home Safety Projects. Available: http://www.rospa.co.uk/hsfacts.htm

[14] Swain AD, Guttman HE (1983) A Handbook of Human Reliability Analysis with Emphasis on Nuclear Power Plant Applications. Nureg/CR-1278 (Washington, DC: USNRC) 
Table 1: Older Adults' choice of domestic activities to be detected by an IDAS

\begin{tabular}{|l|l|l|l|}
\hline No. & Activity to be detected & Yes & Maybe \\
\hline 4 & Personal Temperature Regulation & $\mathrm{X}$ & \\
\hline 5 & House Temperature Regulation & $\mathrm{X}$ & \\
\hline 8 & Activity (Movement/Mobility) & & $\mathrm{X}$ \\
\hline 9 & Management of Existing Medical Conditions & $\mathrm{X}$ & \\
\hline 10 & Management of Sudden Onset Medical Conditions & $\mathrm{X}$ & \\
\hline 11 & Management of Slow Onset Med Conditions & & $\mathrm{X}$ \\
\hline 16 & Home Security (monitor entry \& exits points) & $\mathrm{X}$ & \\
\hline 17 & Personal Security (alarms \& self defence) & $\mathrm{X}$ & \\
\hline 22 & Personal Hygiene (e.g. hair, body, hands, teeth) & & $\mathrm{X}$ \\
\hline
\end{tabular}


Table 2: Careworkers' choice of domestic activities to be detected by an IDAS

\begin{tabular}{|l|l|l|l|}
\hline No. & Activity to be detected & Yes & Maybe \\
\hline 1 & Food/Nutrient Intake & $\mathrm{X}$ & \\
\hline 2 & Drink/Fluid Intake & $\mathrm{X}$ & \\
\hline 3 & Sleep/Rest & $\mathrm{X}$ & \\
\hline 4 & Temperature Regulation (Personal) & $\mathrm{X}$ & \\
\hline 5 & Temperature Regulation (House) & $\mathrm{X}$ & \\
\hline 6 & Elimination (Excretion) & $\mathrm{X}$ & \\
\hline 7 & Elimination (Urination) & $\mathrm{X}$ & \\
\hline 8 & Activity (Movement/Mobility) & & $\mathrm{X}$ \\
\hline 9 & Management of Existing Medical Conditions & $\mathrm{X}$ & \\
\hline 10 & Management of Sudden Onset Medical Conditions & $\mathrm{X}$ & \\
\hline 11 & Management of Slow Onset Med Conditions & $\mathrm{X}$ & \\
\hline 12 & Financial Management & $\mathrm{X}$ & $\mathrm{X}$ \\
\hline 13 & Monitor \& Maintain Furniture, Fixtures \& Fittings & $\mathrm{X}$ & \\
\hline 14 & Monitor \& Maintain Home Appliances & $\mathrm{X}$ & \\
\hline 15 & Monitor \& Maintain Utilities e.g. gas, electricity & $\mathrm{X}$ & \\
\hline 16 & Home Security (monitor entry \& exits points) & $\mathrm{X}$ & \\
\hline 17 & Personal Security (alarms \& self defence) & $\mathrm{X}$ & \\
\hline 18 & Home Entertainment (e.g. TV, Reading, Hobbies) & $\mathrm{X}$ \\
\hline 19 & Outside Home Entertainment (e.g. Outings \& Hobbies) & & $\mathrm{X}$ \\
\hline 20 & Communication & $\mathrm{X}$ \\
\hline 21 & Home Hygiene & & \\
\hline 22 & Personal Hygiene (Hair, body, hands, teeth) & & \\
\hline 23 & Food/Drink Storage & Food/Drink Preparation & \\
\hline 24 & Food/Drink Cooking/Making & & \\
\hline 25 & Clothes Hygiene & & \\
\hline
\end{tabular}

